University of Nebraska - Lincoln

DigitalCommons@University of Nebraska - Lincoln

Faculty Publications from the Department of Electrical \& Computer Engineering, Department Electrical and Computer Engineering

$9-2011$

\title{
Secure Wireless Communication and Optimal Power Control Under Statistical Queueing Constraints
}

Deli Qiao

University of Nebraska-Lincoln, dlqiao@ce.ecnu.edu.cn

M. Cenk Gursoy

University of Nebraska-Lincoln, gursoy@engr.unl.edu

Senem Velipasalar

University of Nebraska-Lincoln, velipasa@engr.unl.edu

Follow this and additional works at: https://digitalcommons.unl.edu/electricalengineeringfacpub

Part of the Electrical and Computer Engineering Commons

Qiao, Deli; Cenk Gursoy, M.; and Velipasalar, Senem, "Secure Wireless Communication and Optimal Power Control Under Statistical Queueing Constraints" (2011). Faculty Publications from the Department of Electrical and Computer Engineering. 204.

https://digitalcommons.unl.edu/electricalengineeringfacpub/204

This Article is brought to you for free and open access by the Electrical \& Computer Engineering, Department of at DigitalCommons@University of Nebraska - Lincoln. It has been accepted for inclusion in Faculty Publications from the Department of Electrical and Computer Engineering by an authorized administrator of DigitalCommons@University of Nebraska - Lincoln. 


\title{
Secure Wireless Communication and Optimal Power Control Under Statistical Queueing Constraints
}

\author{
Deli Qiao, Student Member, IEEE, Mustafa Cenk Gursoy, Member, IEEE, and Senem Velipasalar, Member, IEEE
}

\begin{abstract}
In this paper, secure transmission of information over fading broadcast channels is studied in the presence of statistical queueing constraints. Effective capacity is employed as a performance metric to identify the secure throughput of the system, i.e., effective secure throughput. It is assumed that perfect channel side information (CSI) is available at both the transmitter and the receivers. Initially, the scenario in which the transmitter sends common messages to two receivers and confidential messages to one receiver is considered. For this case, the effective secure throughput region, which is the region of constant arrival rates of common and confidential messages that can be supported by the buffer-constrained transmitter and fading broadcast channel, is defined. It is proven that this effective throughput region is convex implying that time-sharing between any two viable transmission and power control strategies results in effective throughput values inside the region. Then, the optimal power control policies that achieve the boundary points of the effective secure throughput region are investigated and an algorithm for the numerical computation of the optimal power adaptation schemes is provided. Additionally, the throughput region achieved by time-division multiplexing of common and confidential messages is explored. Subsequently, the special case in which the transmitter sends only confidential messages to one receiver is addressed in more detail. For this case, effective secure throughput is formulated and two different power adaptation policies are studied. These power adaptation policies are compared with the opportunistic ones that are optimal in the absence of quality of service (QoS) constraints. It is shown that opportunistic schemes, in which data transmission with high rates and high power occurs only when the main channel is much better than the eavesdropper channel, are no longer optimal under buffer constraints, and the transmitter should send the data at a certain moderate rate and power even when the main channel strength is comparable to that of the eavesdropper channel to avoid buffer overflows.
\end{abstract}

Index Terms-Effective secure throughput, information-theoretic security, optimal power control, quality of service (QoS) constraints, secure broadcasting, superposition coding, time-division multiplexing (TDM).

\section{INTRODUCTION}

$\mathbf{S}$ ECURITY is an important consideration in wireless systems due to the broadcast nature of wireless transmissions. In a pioneering work, Wyner in [1] addressed the security

Manuscript received September 29, 2010; revised March 21, 2011; accepted April 25, 2011. Date of publication May 19, 2011; date of current version August 17,2011 . This work was supported by the National Science Foundation under Grant CNS - 0834753 and Grant CCF - 0917265. This work was presented in part at the the IEEE International Symposium on Information Theory (ISIT), Austin, TX, in June 2010. The associate editor coordinating the review of this manuscript and approving it for publication was Dr. Hisato Iwai.

The authors are with the Department of Electrical Engineering, University of Nebraska-Lincoln, Lincoln, NE68588 USA (e-mail: dqiao726@huskers.unl. edu; gursoy@engr.unl.edu; velipasa@engr.unl.edu).

Color versions of one or more of the figures in this paper are available online at http://ieeexplore.ieee.org.

Digital Object Identifier 10.1109/TIFS.2011.2155650 problem from an information-theoretic point of view and considered a wiretap channel model. He proved that secure transmission of confidential messages to a destination in the presence of a degraded wiretapper can be achieved, and he established the secrecy capacity which is defined as the highest rate of reliable communication from the transmitter to the legitimate receiver while keeping the wiretapper completely ignorant of the transmitted messages. Recently, there have been numerous studies addressing information-theoretic security [2]-[5]. For instance, the impact of fading has been investigated in [2], where it has been shown that a nonzero secrecy capacity can be achieved even when the eavesdropper channel is better than the main channel on average. The secrecy capacity region of the fading broadcast channel with confidential messages and associated optimal power control policies have been identified in [3], where it is shown that the transmitter allocates more power as the strength of the main channel increases with respect to that of the eavesdropper channel.

In addition to security issues, providing acceptable performance and quality is vital to many applications. For instance, voice over IP (VoIP), interactive-video (e.g., video-conferencing), and streaming-video systems are required to satisfy certain buffer or delay constraints, and the recent proliferation and expected widespread use of multimedia applications in next-generation wireless systems call for a rigorous performance analysis under such quality of service (QoS) considerations. A performance measure for these systems is the effective capacity [6], which can be seen as the maximum constant arrival rate that a given time-varying service process can support while satisfying statistical QoS constraints imposed in the form of limitations on the buffer length. Effective capacity is recently studied in various wireless scenarios (see, e.g., [7]-[11] and references therein). For instance, Tang and Zhang in [7] considered the effective capacity when both the receiver and transmitter know the instantaneous channel gains, and derived the optimal power and rate adaptation policies that maximize the system throughput under QoS constraints. Liu et al. in [9] considered fixed-rate transmission schemes and analyzed the effective capacity and related resource requirements for Markov wireless channel models. In [10] and [11], energy efficiency is addressed when the wireless systems operate under buffer constraints and employ either adaptive or fixed transmission schemes.

The above-mentioned studies addressed the physical-layer security and QoS limitations separately. However, the joint treatment of these considerations is of much interest from both practical and theoretical points of view. The practical relevance is through, for instance, the wide range of military and commercial applications and scenarios in which sensitive multimedia 
information needs to be transmitted in a wireless and secure fashion. The theoretical interest is due to the certain tension that arises when both secrecy and buffer limitations are present. For instance, physical-layer security leads to lower transmission rates. Moreover, the optimal performance in wireless scenarios requires opportunistic transmissions in which one has to wait for high-rate transmission until the main channel between the transmitter and the legitimate receiver is much stronger than the eavesdropper's channel. Note that both end-results may cause buffer overflows and packet losses and may be detrimental in buffer/delay constrained systems. Despite these motivating facts, the combination of security and delay/buffer considerations has received only little attention so far. In [12], Liang et al. analyzed the arrival rates supported by a fading wiretap channel and identified the power allocation policies that take into account the queue lengths. In [13], Youssef et al. studied the delay limited secrecy capacity of fading channels. In [14] and [15], we studied the problem of secure communication over fading channels in the presence of statistical QoS constraints. In particular, we considered in [15] a fading broadcast channel and investigated the optimal power control strategies. However, in [15], we employed instantaneous transmission rate expressions which are only upper bounds for systems that perform coding in each frame (in which fading stays constant) rather than over multiple frames. Moreover, we assumed that both common and confidential messages are subject to the same QoS constraints, and addressed only superposition transmission strategies. In this paper, as major extensions to [15], we use more accurate instantaneous rate expressions, consider the more general asymmetric scenario in which different QoS constraints are imposed on different message types, analyze time-division-multiplexing (TDM) strategies, as well as superposition transmissions, and provide new numerical results.

More specifically, we address both physical-layer security issues and buffer limitations in order to identify the key tradeoffs and optimal transmission strategies. We assume that perfect channel side information (CSI) is available at both the transmitter and receivers. We first consider a secure broadcasting scenario in which the transmitter sends common messages to two receivers and confidential messages to one receiver. For this case, we define the effective secure throughput region as the region of common and confidential message arrival rates that can be supported when the transmitter operates under constraints on buffer violation probabilities. Then, we investigate the optimal power allocation policies that achieve points on the boundary of the effective secrecy throughput region. We provide an algorithm to determine the power allocation as a function of the channel states. We also study the performance achieved when the common and confidential message transmissions are time-division multiplexed rather than occurring simultaneously through superposition coding. Subsequently, we provide a more detailed analysis of the special case in which the transmitter sends no common messages. In this case, the broadcast channel with confidential messages is reduced to a wiretap channel. In this scenario, we provide the expression for the effective secure throughput and analyze two types of control policies by adapting the power with respect to both the main and eavesdropper channel conditions and also with respect to only

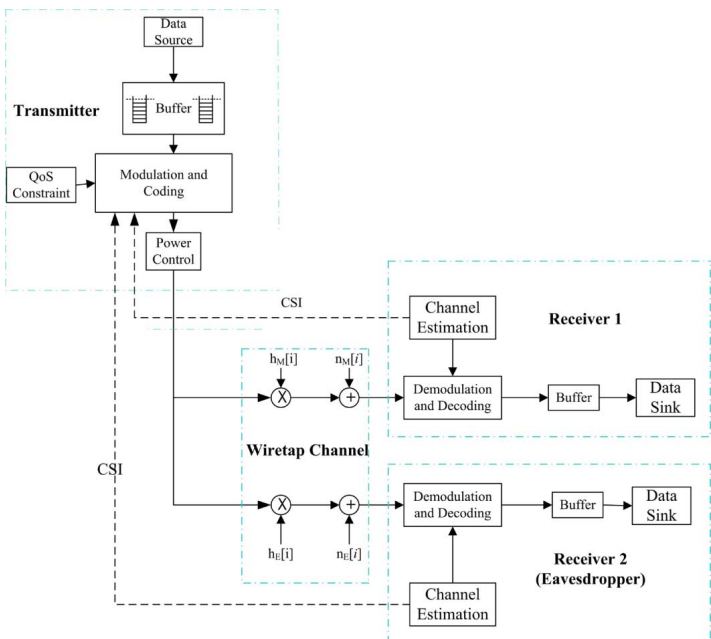

Fig. 1. General system model.

the main channel conditions. Through this analysis, we find that, due to the introduction of the buffer constraints, the transmitter cannot reserve its power for times at which the main channel is much stronger than the eavesdropper channel. Also, we find that adapting the power allocation strategy with respect to both the main and eavesdropper channel CSI rather than only the main channel CSI provides little improvement when QoS constraints become more stringent.

The rest of the paper is organized as follows. Section II briefly describes the system model and the necessary preliminaries on statistical QoS constraints and effective capacity. In Section III, the effective secure throughput region and the corresponding optimal power allocation policies are presented for fading broadcast channels with confidential messages. In Section IV, the special case in which the common message rate is zero is studied in more detail. Finally, Section V concludes the paper.

\section{SYSTEM Model AND PRELIMINARIES}

\section{A. System and Channel Models}

As depicted in Fig. 1, we consider a system with one transmitter and two receivers. We assume that the transmitter sends confidential messages to receiver 1 . From this perspective, receiver 2 can be regarded as an eavesdropper. However, receiver 2 is not necessarily a malicious eavesdropper as we also consider a broadcast scenario in which the transmitter sends common messages to both receivers.

In the model, data sequences generated by the source are divided into frames of duration $T$. These data frames are initially stored in the buffer before they are transmitted over the wireless channel. The channel input-output relationships are given by

and

$$
Y_{1}[i]=h_{1}[i] X[i]+W_{1}[i]
$$

$$
Y_{2}[i]=h_{2}[i] X[i]+W_{2}[i]
$$

where $i$ is the symbol index, $X[i]$ is the channel input in the $i$ th symbol duration, and $Y_{1}[i]$ and $Y_{2}[i]$ represent the channel outputs at receivers 1 and 2, respectively. We assume that $\left\{h_{j}[i], j=1,2\right\}$ 's are jointly stationary and ergodic discrete-time processes, and we denote the magnitude-square 
of the fading coefficients by $z_{j}[i]=\left|h_{j}[i]\right|^{2}$. Considering receiver 1 as the main user, to which we send both the common and confidential messages, and regarding receiver 2 as the eavesdropper for the confidential messages, we replace $z_{1}$ with $z_{M}$ and $z_{2}$ with $z_{E}$ to increase the clarity in the subsequent formulations. The channel input is subject to an average energy constraint $\mathbb{E}\left\{|X[i]|^{2}\right\} \leq \bar{P} / B$ where $B$ is the bandwidth available in the system and hence $\bar{P}$ is the average power constraint (under the assumption that the symbol rate is $B$ complex symbols per second). Above, $W_{j}[i]$ is a zero-mean, circularly symmetric, complex Gaussian random variable with variance $\mathbb{E}\left\{\left|W_{j}[i]\right|^{2}\right\}=N_{j}$. The additive Gaussian noise samples $\left\{W_{j}[i]\right\}$ are assumed to form an independent and identically distributed (i.i.d.) sequence.

We denote the average transmitted signal to noise ratio with respect to receiver 1 as $\mathrm{SNR}=\bar{P} /\left(N_{1} B\right)$. Also, we denote the instantaneous transmit power in the $i$ th frame as $P[i]$. Now, the instantaneous transmitted SNR level for receiver 1 becomes $\mu^{1}[i]=P[i] /\left(N_{1} B\right)$. Then, the average power constraint at the transmitter is equivalent to the average SNR constraint for receiver $1[16]$

$$
\mathbb{E}\left\{\mu^{1}[i]\right\} \leq \mathrm{SNR} .
$$

If we denote the ratio between the noise powers of the two channels as $\gamma=N_{1} / N_{2}$, the instantaneous transmitted SNR level for receiver 2 becomes $\mu^{2}[i]=\gamma \mu^{1}[i]$.

\section{B. Secrecy Capacity Region With Common Messages}

We consider a block-fading channel in which the fading coefficients stay constant for the block duration of $T$ seconds and change independently across the blocks. We assume that both the transmitter and receivers have perfect CSI. Equipped with the channel knowledge, the transmitter employs power control. We denote the power allocation policies for the common and confidential messages by $\mu=\left(\mu_{0}(\mathbf{z}), \mu_{1}(\mathbf{z})\right)$, respectively, where $\mathbf{z}=\left(z_{M}, z_{E}\right)$ is the vector composed of the channel states of receivers 1 and 2 . Note that the power control policies are defined as instantaneous power levels normalized by the noise power $N_{1}$ at receiver 1, i.e., $\mu_{0}(\mathbf{z})=P_{0}[i] /\left(N_{1} B\right)$ and $\mu_{1}(\mathbf{z})=P_{1}[i] /\left(N_{1} B\right)$, where $P_{0}[i]$ and $P_{1}[i]$ are the instantaneous powers of the common and confidential messages, which are in general functions of the fading states z. Since perfect CSI is assumed to be available at the transmitter, the transmitter is able to adapt its transmission power to the instantaneous channel state realization. Note also that this power adaptation should be performed under the average power constraint $\bar{P}$. Hence, we should have $\mathbb{E}_{\mathbf{z}}\left\{P_{0}+P_{1}\right\} \leq \bar{P}$ which, by normalizing all sides with $N_{1} B$, translates to

$$
\mathbb{E}_{\mathbf{z}}\left\{\mu_{0}(\mathbf{z})+\mu_{1}(\mathbf{z})\right\} \leq \mathrm{SNR} .
$$

By considering two different regions of the fading state space, we can further express the constraint in (4) as

$$
\mathbb{E}_{\mathbf{z} \in \mathcal{Z}}\left\{\mu_{0}(\mathbf{z})+\mu_{1}(\mathbf{z})\right\}+\mathbb{E}_{\mathbf{z} \in \mathcal{Z}^{c}}\left\{\mu_{0}(\mathbf{z})\right\} \leq \mathrm{SNR}
$$

where we have defined the region $\mathcal{Z}$ as $\mathcal{Z}=$ $\left\{\mathbf{z} \geq 0: z_{M}>\gamma z_{E}\right\}$ and its complement in the first quadrant as $\mathcal{Z}^{c}=\left\{\mathbf{z} \geq 0: z_{M} \leq \gamma z_{E}\right\}$. Equation (5) follows from the fact that in region $\mathcal{Z}^{c}$, the eavesdropper's channel is stronger than the main channel and consequently instantaneous secrecy capacity is zero. Hence, when $\mathbf{z} \in \mathcal{Z}^{c}$, confidential messages should not be transmitted and we have to set $\mu_{1}(\mathbf{z})=0$. Following the above definitions, we finally define $\mathcal{U}$ as set of the power allocation policies that satisfy the average SNR constraint, i.e.,

$$
\begin{aligned}
\mathcal{U}=\left\{\mu: \mathbb{E}_{\mathbf{z} \in \mathcal{Z}}\left\{\mu_{0}(\mathbf{z})+\mu_{1}(\mathbf{z})\right\}\right. & \\
& \left.+\mathbb{E}_{\mathbf{z} \in \mathcal{Z}^{c}}\left\{\mu_{0}(\mathbf{z})\right\} \leq \mathrm{SNR}=\frac{\bar{P}}{N_{1} B}\right\} .
\end{aligned}
$$

With the above power control policies, the maximum instantaneous common message rate in each block with power control policy $\mu$ is given by $[3, \mathrm{Sec} . \mathrm{V}]$

$$
R_{0}= \begin{cases}\log _{2}\left(1+\frac{\gamma \mu_{0}(\mathbf{z}) z_{E}}{1+\gamma \mu_{1}(\mathbf{z}) z_{E}}\right), & \mathbf{z} \in \mathcal{Z} \\ \log _{2}\left(1+\mu_{0}(\mathbf{z}) z_{M}\right), & \mathbf{z} \in \mathcal{Z}^{c}\end{cases}
$$

under the assumption that channel coding is performed in each block of duration $T$ seconds, and the block length, which is $T B$ symbols, is large enough so that the probability of error is negligible and hence communication at these rates is reliable. Under similar assumptions, the maximum instantaneous confidential message rate is given by

$$
R_{1}= \begin{cases}\log _{2}\left(1+\mu_{1}(\mathbf{z}) z_{M}\right)-\log _{2}\left(1+\gamma \mu_{1}(\mathbf{z}) z_{E}\right), & \mathbf{z} \in \mathcal{Z} \\ 0, & \mathbf{z} \in \mathcal{Z}^{c}\end{cases}
$$

Then, the ergodic secrecy capacity region for the fading broadcast channel with common and confidential messages is ${ }^{1}$

$$
\mathcal{C}_{s}=\bigcup_{\mu \in \mathcal{U}}\left\{\begin{array}{c}
\left(R_{0, \text { avg }}, R_{1, \text { avg }}\right): \\
R_{0, \text { avg }} \leq \mathbb{E}_{\mathbf{z} \in \mathcal{Z}}\left\{\log _{2}\left(1+\frac{\gamma \mu_{0}(\mathbf{z}) z_{E}}{1+\gamma \mu_{1}(\mathbf{z}) z_{E}}\right)\right\} \\
+\mathbb{E}_{\mathbf{z} \in \mathcal{Z}_{c}}\left\{\log _{2}\left(1+\mu_{0}(\mathbf{z}) z_{M}\right)\right\} \\
R_{1, \text { avg }} \leq \mathbb{E}_{\mathbf{z} \in \mathcal{Z}}\left\{\log _{2}\left(1+\mu_{1}(\mathbf{z}) z_{M}\right)\right. \\
\left.-\log _{2}\left(1+\gamma \mu_{1}(\mathbf{z}) z_{E}\right)\right\}
\end{array}\right\} .
$$

When $\mathbf{z} \in \mathcal{Z}$, the common and confidential messages are sent simultaneously by using superposition encoding methods. Moreover, we note that coding schemes for accomplishing secrecy in the physical layer mainly employ random binning schemes and more details can be found in [1]-[5].

The following result shows the convexity of the above capacity region.

Proposition 1: The ergodic secrecy capacity region $\mathcal{C}_{s}$ is convex.

Proof: Let $\mathbf{R}=\left(R_{0, \text { avg }}, R_{1, \text { avg }}\right) \in \mathcal{C}_{s}$ and $\mathbf{R}^{\prime}=$ $\left(R_{0, \text { avg }}^{\prime}, R_{1, \text { avg }}^{\prime}\right) \in \mathcal{C}_{s}$ be two rate pairs achieved by power control policies $\mu=\left(\mu_{0}(\mathbf{z}), \mu_{1}(\mathbf{z})\right) \in \mathcal{U}$ and $\mu^{\prime}=\left(\mu_{0}^{\prime}(\mathbf{z}), \mu_{1}^{\prime}(\mathbf{z})\right) \in \mathcal{U}$, respectively. Now, assume that a time-sharing strategy is employed, and power control policy $\mu$ is used $\alpha \in(0,1)$ fraction of the time and $\mu^{\prime}$ is employed in the remaining $1-\alpha$ fraction of the time. The new power control policy can be expressed as

$$
\mu^{*}= \begin{cases}\mu, & \alpha \text { fraction of the time } \\ \mu^{\prime}, & 1-\alpha \text { fraction of the time. }\end{cases}
$$

\footnotetext{
${ }^{1}$ Note that if coding over all channel states is allowed, a larger capacity region can be achieved (see, e.g., [3, Sec. IV]).
} 
Since $\mu \in \mathcal{U}$ and $\mu^{\prime} \in \mathcal{U}$, we can easily see that $\mathbb{E}\left\{\mu^{*}\right\}=$ $\alpha \mathbb{E}\{\mu\}+(1-\alpha) \mathbb{E}\left\{\mu^{\prime}\right\} \leq$ SNR, and hence $\mu^{*} \in \mathcal{U}$ as well. Moreover, this time-sharing strategy achieves $\alpha \mathbf{R}+(1-\alpha) \mathbf{R}^{\prime}$ with the power control policy $\mu^{*}$. Therefore, we conclude that $\alpha \mathbf{R}+(1-\alpha) \mathbf{R}^{\prime} \in \mathcal{C}_{s}$, showing the convexity of $\mathcal{C}_{s}$.

\section{Statistical QoS Constraints and Effective Secure Throughput}

In [6], effective capacity is defined as the maximum constant arrival rate ${ }^{2}$ that a given service process can support in order to guarantee a statistical QoS requirement specified by the QoS exponent $\theta$. If we define $Q$ as the stationary queue length, then $\theta$ is the decay rate of the tail distribution of the queue length $Q$

$$
\lim _{q \rightarrow \infty} \frac{\log P(Q \geq q)}{q}=-\theta .
$$

Therefore, for large $q_{\max }$, we have the following approximation for the buffer violation probability: $P\left(Q \geq q_{\max }\right) \approx e^{-\theta q_{\max }}$. Hence, while larger $\theta$ corresponds to more strict QoS constraints, smaller $\theta$ implies looser QoS guarantees. Similarly, if $D$ denotes the steady-state delay experienced in the buffer, then $P\left(D \geq d_{\max }\right) \approx e^{-\theta \delta d_{\max }}$ for large $d_{\max }$, where $\delta$ is determined by the arrival and service processes [8].

The effective capacity is given by

$$
C(\theta)=-\lim _{t \rightarrow \infty} \frac{1}{\theta t} \log _{e} \mathbb{E}\left\{e^{-\theta S[t]}\right\} \quad \text { bits/s }
$$

where the expectation is with respect to $S[t]=\sum_{i=1}^{t} s[i]$, which is the time-accumulated service process. $\{s[i], i=$ $1,2, \ldots\}$ denote the discrete-time stationary and ergodic stochastic service process. We define the effective capacity obtained when the service rate is confined by the secrecy capacity region as the effective secure throughput.

Under the block fading assumption, the service rate in the $i$ th block is $s[i]=T B R$ bits per $T$ seconds, where $R$ is the instantaneous service rate for either common or confidential messages, and $B$ is the bandwidth. Note that $s[i]$ varies independently from one block to another due to the block fading assumption. Then, (12) can be written as

$$
C(\theta)=-\frac{1}{\theta T} \log _{e} \mathbb{E}_{\mathbf{z}}\left\{e^{-\theta T B R}\right\} \quad \text { bits } / \mathrm{s} .
$$

Above, if $R$ is equal to $R_{0}$ in (7), then $C(\theta)$ is the maximum effective capacity (or equivalently the maximum constant arrival rate) of the common messages, which can be achieved with the power allocation policy $\mu=\left(\mu_{0}, \mu_{1}\right)$ under queueing constraints specified by the QoS exponent $\theta$. Similarly, if $R=R_{1}$ in (8), then $C(\theta)$ is the maximum effective capacity of the confidential messages achieved with the power control policy $\mu$. Note that in both cases, $R$ depends on the fading states $\mathbf{z}$, and the expectation in (13) is with respect to $\mathbf{z}$.

Finally, we denote the effective capacity normalized by bandwidth $B$ as

$$
\mathrm{C}(\theta)=\frac{C(\theta)}{B} \quad \text { bits } / \mathrm{s} / \mathrm{Hz}
$$

${ }^{2}$ For time-varying arrival rates, effective capacity specifies the effective bandwidth of the arrival process that can be supported by the channel.

\section{EfFective Secure Throughput Region With Common Messages ANd Optimal POWER CONTROL}

In this section, we investigate the secure throughput region of and the optimal power control policies for the fading broadcast channel with confidential messages (BCC) in the presence of statistical QoS constraints. Hence, in the considered scenario, the transmitter sends common messages to two receivers, sends confidential messages to only one receiver, and operates under buffer constraints. Liang et al. in [3] showed that the fading channel can be viewed as a set of parallel subchannels with each subchannel corresponding to one fading state. Subsequently, the ergodic secrecy capacity region is determined and the optimal power allocation policies achieving the boundary of the capacity region are identified in [3]. Outage performance is also studied for cases in which long transmission delays cannot be tolerated and coding and decoding needs to be performed in one block.

\section{A. Effective Secure Throughput Region}

In this paper, we analyze the performance under statistical buffer constraints by considering the effective capacity formulation. We assume that common and confidential message transmissions are subject to individual statistical QoS constraints described by $\theta_{0}$ and $\theta_{1}$, respectively. Using the effective capacity expression in (13), we first have the following definition for the effective secure throughput region.

Definition 1: The effective secure throughput region of the fading BCC is

$$
\begin{aligned}
\mathcal{C}_{e s}=\bigcup_{\substack{\mathbf{R}=\left(R_{0}, R_{1}\right) \\
\text { s.t. } \mathbb{E}\{\mathbf{R}\} \in \mathcal{C}_{\mathbf{s}}}}\left\{\left(\mathrm{C}_{0}, \mathrm{C}_{1}\right): \mathrm{C}_{j}\right. \\
\left.\quad \leq-\frac{1}{\theta_{j} T B} \log _{e} \mathbb{E}\left\{e^{-\theta_{j} T B R_{j}}\right\}, j=0,1\right\}
\end{aligned}
$$

where $\mathbf{R}=\left(R_{0}, R_{1}\right)$ is the vector composed of the instantaneous rates for the common and confidential messages, respectively.

Note that the union in (15) is over the distributions of the vector $\mathbf{R}$ such that the expected value $\mathbb{E}\{\mathbf{R}\}$ lies in the ergodic secrecy capacity region $\mathcal{C}_{s}$. Note also that the maximum values of the instantaneous rates $R_{0}$ and $R_{1}$ for a given power control policy $\mu$ are provided by (7) and (8). Moreover, in (15), $C_{0}$ and $C_{1}$ denote the effective capacities of common and confidential messages, respectively.

Since the ergodic secrecy capacity region is convex as proved in Proposition 1, we can easily prove the following.

Theorem 1: The effective secrecy throughput region $\mathcal{C}_{e s}$ defined in (15) is convex.

Proof: Let the two effective capacity pairs $\underline{\mathrm{C}}=\left(\mathrm{C}_{0}, \mathrm{C}_{1}\right)$ and ${\underline{C^{\prime}}}^{\prime}=\left(\mathrm{C}_{0}^{\prime}, \mathrm{C}_{1}^{\prime}\right)$ belong to $\mathcal{C}_{e s}$. Therefore, there exist some $\mathbf{R}=\left(R_{0}, R_{1}\right)$ and $\mathbf{R}^{\prime}=\left(R_{0}^{\prime}, R_{1}^{\prime}\right)$ for $\underline{\mathrm{C}}$ and $\underline{\mathrm{C}}^{\prime}$, respectively. By a time sharing strategy, for any $\alpha \in(0,1)$, we know that 
$\mathbb{E}\left\{\alpha \mathbf{R}+(1-\alpha) \mathbf{R}^{\prime}\right\} \in \mathcal{C}_{s}$. Denote $\Theta=\left(\theta_{0}, \theta_{1}\right)$. Then, we can write

$$
\begin{aligned}
\alpha \underline{\mathrm{C}} & +(1-\alpha) \underline{\mathrm{C}}^{\prime} \\
= & -\frac{1}{\Theta T B} \log _{e}\left(\mathbb{E}\left\{e^{-\Theta T B \mathbf{R}}\right\}\right)^{\alpha}\left(\mathbb{E}\left\{e^{-\Theta T B \mathbf{R}^{\prime}}\right\}\right)^{1-\alpha} \\
= & -\frac{1}{\Theta T B} \log _{e}\left(\mathbb{E}\left\{\left(e^{-\Theta T B \alpha \mathbf{R}}\right)^{1 / \alpha}\right\}\right)^{\alpha} \\
& \times\left(\mathbb{E}\left\{\left(e^{-\Theta T B(1-\alpha) \mathbf{R}^{\prime}}\right)^{1 /(1-\alpha)}\right\}\right)^{1-\alpha} \\
\leq & -\frac{1}{\Theta T B} \log _{e} \mathbb{E}\left\{e^{-\Theta T B\left(\alpha \mathbf{R}+(1-\alpha) \mathbf{R}^{\prime}\right)}\right\}
\end{aligned}
$$

Above, in (16) - (18), all algebraic operations are with respect to each component of the vectors. For instance, the expression in (16) denotes a vector whose components are $\left\{\left(1 / \theta_{j} T B\right) \log _{e}\left(\mathbb{E}\left\{e^{-\theta_{j} T B R_{j}}\right\}\right)^{\alpha}\left(\mathbb{E}\left\{e^{-\theta_{j} T B R_{j}^{\prime}}\right\}\right)^{1-\alpha}\right\}$ for $j=0,1$. The inequality in (18) follows from Hölder's inequality. Hence, $\alpha \underline{\mathrm{C}}+(1-\alpha) \underline{\mathrm{C}}^{\prime}$ lies in the throughput region, showing the convexity.

Due to the convexity property, the points on the boundary surface of the effective throughput region $\left(\mathrm{C}_{0}^{*}, \mathrm{C}_{1}^{*}\right)$ can be obtained by solving the following optimization problem:

$$
\max _{\mu \in \mathcal{U}} \lambda_{0} \mathrm{C}_{0}+\lambda_{1} \mathrm{C}_{1}
$$

where $\lambda=\left(\lambda_{0}, \lambda_{1}\right)$ is any vector in $\mathfrak{R}_{+}^{2}$, and $\mathrm{C}_{0}$ and $\mathrm{C}_{1}$ are the maximum effective capacity values for a given power control policy $\mu$, i.e., they are the effective capacity values when the instantaneous service rates are the ones given in (7) and (8).

\section{B. Optimal Power Control}

Having characterized the effective secure throughput region, we turn our attention to optimal power control. Note that due to the introduction of QoS constraints, the maximization is over the effective capacities while the service rates are limited by the instantaneous channel capacities.

Next, we derive the optimality conditions for the optimal power allocation $\mu^{*}$ that solves (19). As also provided in Section II-B, the maximal instantaneous common message rate for a given power control policy $\mu$ is

$$
R_{0}= \begin{cases}\log _{2}\left(1+\frac{\gamma \mu_{0}(\mathbf{z}) z_{E}}{1+\gamma \mu_{1}(\mathbf{z}) z_{E}}\right), & \mathbf{z} \in \mathcal{Z} \\ \log _{2}\left(1+\mu_{0}(\mathbf{z}) z_{M}\right), & \mathbf{z} \in \mathcal{Z}^{c}\end{cases}
$$

Similarly, the maximal instantaneous confidential message (or equivalently secrecy) rate is

$$
R_{1}= \begin{cases}\log _{2}\left(\frac{1+\mu_{1}(\mathbf{z}) z_{M}}{1+\gamma \mu_{1}(\mathbf{z}) z_{E}}\right), & \mathbf{z} \in \mathcal{Z} \\ 0, & \mathbf{z} \in \mathcal{Z}^{c} .\end{cases}
$$

Now, using these instantaneous service rates $R_{0}$ and $R_{1}$ in the effective capacity expressions and recalling the average SNR constraint in (6), we can express the Lagrangian of the convex optimization problem in (19) as shown in (22), at the bottom of the page, where $\beta_{j}=\theta_{j} T B / \log _{e} 2, j=0,1, p_{\mathbf{z}}\left(z_{M}, z_{E}\right)$ is the joint distribution function of the fading states $\mathbf{z}=\left(z_{M}, z_{E}\right)$, and $\kappa \geq 0$ is the Lagrange multiplier. Next, we define $\left(\phi_{0}, \phi_{1}\right)$ as

$$
\begin{aligned}
\phi_{0}=\int_{\mathbf{z} \in \mathcal{Z}} & \left(1+\frac{\gamma \mu_{0}(\mathbf{z}) z_{E}}{1+\gamma \mu_{1}(\mathbf{z}) z_{E}}\right)^{-\beta_{0}} p_{\mathbf{z}}\left(z_{M}, z_{E}\right) d \mathbf{z} \\
& +\int_{\mathbf{z} \in \mathcal{Z}^{c}}\left(1+\mu_{0}(\mathbf{z}) z_{M}\right)^{-\beta_{0}} p_{\mathbf{z}}\left(z_{M}, z_{E}\right) d \mathbf{z}
\end{aligned}
$$

and

$$
\begin{aligned}
\phi_{1}=\int_{\mathbf{z} \in \mathcal{Z}}\left(\frac{1+\mu_{1}(\mathbf{z}) z_{M}}{1+\gamma \mu_{1}(\mathbf{z}) z_{E}}\right)^{-\beta_{1}} & p_{\mathbf{z}}\left(z_{M}, z_{E}\right) d \mathbf{z} \\
& +\int_{\mathbf{z} \in \mathcal{Z}^{c}} p_{\mathbf{z}}\left(z_{M}, z_{E}\right) d \mathbf{z}
\end{aligned}
$$

Below, we derive the optimality conditions (that the optimal power control policies should satisfy) by differentiating the Lagrangian with respect to $\mu_{0}$ in regions $\mathcal{Z}^{c}$ and $\mathcal{Z}$ and with respect to $\mu_{1}$ in $\mathcal{Z}$, and making the derivatives equal to zero

$$
\begin{aligned}
\text { 1) } & \frac{\lambda_{0}}{\phi_{0} \log _{e} 2}\left(1+\mu_{0} z_{M}\right)^{-\beta_{0}-1} z_{M}-\kappa \\
& =0 \quad \forall \mathbf{z} \in \mathcal{Z}^{c} \\
\text { 2) } & \frac{\lambda_{0}}{\phi_{0} \log _{e} 2}\left(1+\frac{\gamma \mu_{0} z_{E}}{1+\gamma \mu_{1} z_{E}}\right)^{-\beta_{0}-1} \frac{\gamma z_{E}}{1+\gamma \mu_{1} z_{E}}-\kappa \\
& =0 \quad \forall \mathbf{z} \in \mathcal{Z} \\
3) & -\frac{\lambda_{0}}{\phi_{0} \log _{e} 2}\left(1+\frac{\gamma \mu_{0} z_{E}}{1+\gamma \mu_{1} z_{E}}\right)^{-\beta_{0}-1} \frac{\mu_{0}\left(\gamma z_{E}\right)^{2}}{\left(1+\gamma \mu_{1} z_{E}\right)^{2}} \\
& +\frac{\lambda_{1}}{\phi_{1} \log _{e} 2}\left(\frac{1+\mu_{1} z_{M}}{1+\gamma \mu_{1} z_{E}}\right)^{-\beta_{1}-1} \frac{z_{M}-\gamma z_{E}}{\left(1+\gamma \mu_{1} z_{E}\right)^{2}}-\kappa \\
& =0 \quad \forall \mathbf{z} \in \mathcal{Z}
\end{aligned}
$$

where (25)-(27) are obtained by evaluating the derivative of $\mathcal{J}$ with respect to $\mu_{0}$ when $\mathbf{z} \in \mathcal{Z}^{c}, \mu_{0}$ when $\mathbf{z} \in \mathcal{Z}$, and $\mu_{1}$ when $\mathbf{z} \in \mathcal{Z}$, respectively. Whenever $\mu_{0}$ or $\mu_{1}$ turn out to have negative values through these equations, they are set to 0 due to the convexity of the optimization problem [17].

$$
\begin{aligned}
\mathcal{J}= & -\frac{\lambda_{0}}{\beta_{0} \log _{e} 2} \log _{e}\left(\int_{\mathbf{z} \in \mathcal{Z}}\left(1+\frac{\gamma \mu_{0}(\mathbf{z}) z_{E}}{1+\gamma \mu_{1}(\mathbf{z}) z_{E}}\right)^{-\beta_{0}} p_{\mathbf{z}}\left(z_{M}, z_{E}\right) d \mathbf{z}+\int_{\mathbf{z} \in \mathcal{Z}^{c}}\left(1+\mu_{0}(\mathbf{z}) z_{M}\right)^{-\beta_{0}} p_{\mathbf{z}}\left(z_{M}, z_{E}\right) d \mathbf{z}\right) \\
& -\frac{\lambda_{1}}{\beta_{1} \log _{e} 2} \log _{e}\left(\int_{\mathbf{z} \in \mathcal{Z}}\left(\frac{1+\mu_{1}(\mathbf{z}) z_{M}}{1+\gamma \mu_{1}(\mathbf{z}) z_{M}}\right)^{-\beta_{1}} p_{\mathbf{z}}\left(z_{M}, z_{E}\right) d \mathbf{z}+\int_{\mathbf{z} \in \mathcal{Z}^{c}} p_{\mathbf{z}}\left(z_{M}, z_{E}\right) d \mathbf{z}\right) \\
& -\kappa\left(\mathbb{E}_{\mathbf{z} \in \mathcal{Z}}\left\{\mu_{0}(\mathbf{z})+\mu_{1}(\mathbf{z})\right\}+\mathbb{E}_{\mathbf{z} \in \mathcal{Z}^{c}}\left\{\mu_{0}(\mathbf{z})\right\}\right)
\end{aligned}
$$


We immediately note from (25) that when $\mathbf{z} \in \mathcal{Z}^{c}$ (i.e., when $z_{M} \leq \gamma z_{E}$ and no confidential messages are transmitted), the optimal power control policy for the common messages can be expressed as

$$
\mu_{0}=\left[\frac{1}{\alpha_{1}^{1 /\left(\beta_{0}+1\right)} z_{M}^{\beta_{0} /\left(\beta_{0}+1\right)}}-\frac{1}{z_{M}}\right]^{+} \quad \forall \mathbf{z} \in \mathcal{Z}^{c}
$$

where $\alpha_{1}=\kappa \phi_{0} \log _{e} 2 / \lambda_{0}$. Also, when $\mathbf{z} \in \mathcal{Z}$, if no power is allocated for confidential messages and hence $\mu_{1}=0$, then we see from (26) that we can write the optimal $\mu_{0}$ as

$$
\mu_{0}=\left[\frac{1}{\alpha_{1}^{1 /\left(\beta_{0}+1\right)}\left(\gamma z_{E}\right)^{\beta_{0} /\left(\beta_{0}+1\right)}}-\frac{1}{\gamma z_{E}}\right]^{+} \quad \forall \mathbf{z} \in \mathcal{Z} .
$$

A final remark about $\mu_{0}$ is the following. Noting that the term $\left(1+\gamma \mu_{0} z_{E} /\left(1+\gamma \mu_{1} z_{E}\right)\right)^{-\beta_{0}-1}$ in $(26)$ is less than 1 for $\mu_{0}>$ 0 , we have $\left(\lambda_{0} / \phi_{0} \log _{e} 2\right)\left(\gamma z_{E} /\left(1+\gamma \mu_{1} z_{E}\right)\right)>\kappa$ when $\mu_{0}>$ 0 . Equivalently, having $\left(\lambda_{0} / \phi_{0} \log _{e} 2\right)\left(\gamma z_{E} /\left(1+\gamma \mu_{1} z_{E}\right)\right) \leq$ $\kappa$ implies that $\mu_{0}=0$.

Regarding the optimal power control for the confidential messages, we have the following observations from the optimality conditions. We remark that when $\mu_{0}=0,(27)$ becomes

$$
\frac{\lambda_{1}}{\phi_{1} \log _{e} 2}\left(\frac{1+\mu_{1} z_{M}}{1+\gamma \mu_{1} z_{E}}\right)^{-\beta_{1}-1} \frac{z_{M}-\gamma z_{E}}{\left(1+\gamma \mu_{1} z_{E}\right)^{2}}-\kappa=0
$$

from which the optimal $\mu_{1}$ can be computed.

When we have both $\mu_{0}>0$ and $\mu_{1}>0$, the optimal power allocations can be obtained by solving (26) and (27) simultaneously. In this case, a certain condition that depends only on $\mu_{1}$ can be obtained. By combining the equations in (26) and (27) and applying several straightforward algebraic manipulations, we get

$$
\begin{aligned}
& \frac{\lambda_{1}}{\kappa \phi_{1} \log _{e} 2}(\left.\frac{1+\mu_{1} z_{M}}{1+\gamma \mu_{1} z_{E}}\right)^{-\beta_{1}-1} \frac{z_{M}-\gamma z_{E}}{\left(1+\gamma \mu_{1} z_{E}\right)^{2}} \\
&-\left(\frac{\lambda_{0}}{\kappa \phi_{0} \log _{e} 2} \frac{\gamma z_{E}}{1+\gamma \mu_{1} z_{E}}\right)^{1 /\left(\beta_{0}+1\right)}=0
\end{aligned}
$$

which depends only on $\mu_{1}$. The positive solution $\mu_{1}>0$ of this equation provides the optimal power control policy for the confidential messages. Once optimal $\mu_{1}$ is determined, the optimal policy $\mu_{0}$ can be easily found from (26).

As seen in the above discussion, we have closed-form expressions for the optimal power control policy for the common messages in special cases (e.g., when $\mathbf{z} \in \mathcal{Z}^{c}$ or when $\mu_{1}=0$ ). On the other hand, the optimal power control policy for the confidential messages does not assume simple closed-form formulas even in special cases. Hence, optimal power control is in general determined through numerical computations. Making use of the optimality conditions in (25)-(27) and the characterizations in (28) through (31), we propose the following algorithm below to obtain the optimal power adaptation policies. This algorithm is used in the numerical results presented in Section III-D.

\section{AlgORITHM PC (Power Control)}

1 Given $\lambda_{0}, \lambda_{1}$, initialize $\phi_{0}, \phi_{1}$;
Initialize $\kappa$;

Determine $\alpha_{1}=\kappa \phi_{0} \log _{e} 2 / \lambda_{0}, \alpha_{2}=\kappa \phi_{1} \log _{e} 2 / \lambda_{1}$;

if $z_{M}-\gamma z_{E}>0$

then if $z_{M}-\gamma z_{E}>\alpha_{2}$

then Compute $\mu_{1}$ from (30);

if $\mu_{1}>1 / \alpha_{1}-1 / \gamma z_{E}$ or $\gamma z_{E}<\alpha_{1}$

then $\mu_{0}=0$;

else if (31) returns positive solution

thenCompute $\mu_{0}$ and $\mu_{1}$ from (26) and (27);

else $\mu_{1}=0$,

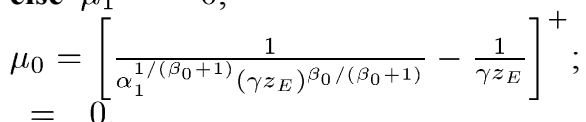

else $\mu_{1}=0$,

$$
\mu_{0}=\left[\frac{1}{\alpha_{1}^{1 /\left(\beta_{0}+1\right)}\left(\gamma z_{E}\right)^{\beta_{0} /\left(\beta_{0}+1\right)}}-\frac{1}{\gamma z_{E}}\right]^{+} ;
$$

else $\mu_{1}=0, \mu_{0}=\left[\frac{1}{\alpha_{1}^{1 /\left(\beta_{0}+1\right)} z_{M}^{\beta_{0} /\left(\beta_{0}+1\right)}}-\frac{1}{z_{M}}\right]^{+}$;

15 Check if the obtained $\mu_{0}$ and $\mu_{1}$ satisfy the average

16 power constraint with equality;

17 if not satisfied with equality

18 then update the value of $\kappa$ and return to Step 3;

19 else move to Step 20;

20 Evaluate $\phi_{0}$ and $\phi_{1}$ with the obtained power control policies;

21 Check if the new values of $\phi_{0}$ and $\phi_{1}$ agree (up to

22 a certain margin) with those used in Step 3;

23 if do not agree

24 then update the values of $\phi_{0}$ and $\phi_{1}$ and return to Step 2;

25 else declare the obtained power allocation policies $\mu_{1}$

26 and $\mu_{2}$ as the optimal ones.

\section{Time-Division Multiplexing (TDM) of Common and Confidential Messages}

In previous sections, we have implicitly assumed that when $\mathbf{z} \in \mathcal{Z}$, common and confidential messages are transmitted simultaneously by using superposition coding techniques. Subsequently, receiver 1 is assumed to perform successive decoding. Hence, receiver 1 initially decodes the common message treating the codeword for the confidential message as noise and then subtracts the decoded common message from the signal and decodes the confidential message in the absence of any interference. These operations are considered due to their information-theoretic optimality. However, superposition coding at the transmitter and successive decoding at the receiver may lead to difficulties in implementation. Due to this, orthogonal transmissions are generally preferred in practical communication systems. With this motivation, we in this section investigate the effective secrecy throughput and the optimal power control policies in the scenario where common and confidential messages are transmitted in different time intervals through a TDM scheme.

Here, we assume that when $\mathbf{z} \in \mathcal{Z}$, a fixed fraction $\tau \in[0,1]$ of each block is allocated to the confidential message transmission, and the remaining $1-\tau$ fraction of the block is allocated to the common message. Note that as before, when $\mathbf{z} \in \mathcal{Z}^{c}$, only 
the common message is transmitted. Thus, the transmission rate for confidential messages is now

$$
R_{1}= \begin{cases}\tau\left[\log _{2}\left(1+\mu_{1}(\mathbf{z}) z_{M}\right)-\log _{2}\left(1+\gamma \mu_{1}(\mathbf{z}) z_{E}\right)\right], & \mathbf{z} \in \mathcal{Z} \\ 0, & \mathbf{z} \in \mathcal{Z}^{c}\end{cases}
$$

and the transmission rate for common messages is

$$
R_{0}= \begin{cases}(1-\tau) \log _{2}\left(1+\gamma \mu_{0}(\mathbf{z}) z_{E}\right), & \mathbf{z} \in \mathcal{Z} \\ \log _{2}\left(1+\mu_{0}(\mathbf{z}) z_{M}\right), & \mathbf{z} \in \mathcal{Z}^{c}\end{cases}
$$

where $\mu=\left(\mu_{0}(\mathbf{z}), \mu_{1}(\mathbf{z})\right) \in \mathcal{U}$.

Now, substituting (32) and (33) into (13) and normalizing the effective rate with the bandwidth $B$ give us the effective capacity for common messages as

$$
\begin{array}{r}
\mathrm{C}_{0}=-\frac{1}{\beta_{0} \log _{e} 2} \log _{e}\left(\mathbb{E}_{\mathbf{z} \in \mathcal{Z}\left\{\left(1+\gamma \mu_{0}(\mathbf{z}) z_{E}\right)^{-(1-\tau) \beta_{0}}\right\}}\right. \\
\left.+\mathbb{E}_{\mathbf{z} \in \mathcal{Z}^{c}}\left\{\left(1+\mu_{0}(\mathbf{z}) z_{M}\right)^{-\beta_{0}}\right\}\right)
\end{array}
$$

and the effective capacity for confidential messages as

$$
\begin{aligned}
& \mathrm{C}_{1}=-\frac{1}{\beta_{1} \log _{e} 2} \\
& \times \log _{e}\left(\mathbb{E}_{\mathbf{z} \in \mathcal{Z}}\left\{\left(\frac{1+\mu_{1}(\mathbf{z}) z_{M}}{1+\gamma \mu_{1}(\mathbf{z}) z_{E}}\right)^{-\tau \beta_{1}}\right\}+\mathbb{E}_{\mathbf{z} \in \mathcal{Z}^{c}}\{1\}\right)
\end{aligned}
$$

where we again have $\beta_{j}=\theta_{j} T B / \log _{e} 2, j=0,1$. Above, we see that through the use of this TDM scheme, we have $C_{0}$ depend only on $\mu_{0}$ and $C_{1}$ depend only on $\mu_{1}$. At the same time, note that we still have the constraint $\mathbb{E}_{\mathbf{z}}\left\{\mu_{0}(\mathbf{z})+\mu_{1}(\mathbf{z})\right\} \leq$ SNR.

In order to identify the effective throughput region achieved with TDM, we employ the following two steps. We initially decouple the problem of finding the optimal power control policies $\mu_{0}$ and $\mu_{1}$ by fixing the average power and time allocated to the transmission of common and confidential messages. Subsequently, we identify the effective capacities achieved with different average power and time allocations.

1) Assume that $\mathbb{E}_{\mathbf{z}}\left\{\mu_{1}(\mathbf{z})\right\}=\rho \mathrm{SNR}$ and $\mathbb{E}_{\mathbf{z}}\left\{\mu_{0}(\mathbf{z})\right\}=(1-$ $\rho)$ SNR for some $\rho \in[0,1]$. For fixed $\tau$ and $\rho$, the optimal power control policies can be found by solving the following two independent optimization problems:

$$
\mathrm{C}_{0}^{*}=\max _{\substack{\mu_{0}(\mathbf{z}) \\ \mathrm{C}_{1}^{*}}} \mathrm{C}_{0}
$$

where $C_{0}$ and $C_{1}$ are given by (34) and (35).
Using the Lagrangian method to solve the problems in (36) and (37) yields us the optimal power control policies for common and confidential message transmissions, respectively. The optimal $\mu_{1}^{*}(\mathbf{z})$ can be obtained from the following equation:

$$
\begin{aligned}
\frac{\partial \mathcal{J}}{\partial \mu_{1}\left(z_{M}, z_{E}\right)}=\lambda-\beta_{1} \tau & \left(\frac{1+\mu_{1} z_{M}}{1+\gamma \mu_{1} z_{E}}\right)^{-\beta_{1} \tau} \\
\times & \frac{z_{M}-\gamma z_{E}}{\left(1+\mu_{1} z_{M}\right)\left(1+\gamma \mu_{1} z_{E}\right)}=0
\end{aligned}
$$

where $\lambda$ is the Lagrange multiplier whose value is chosen to satisfy the average power constraint $\mathbb{E}\left\{\mu_{1}(\mathbf{z})\right\} \leq \rho \mathrm{SNR}$.

The optimal $\mu_{0}^{*}(\mathbf{z})$ is given by (39), shown at the bottom of the page, where $\alpha_{0}$ is chosen to satisfy the average power constraint with equality $\mathbb{E}_{\mathbf{z}}\left\{\mu_{0}^{*}(\mathbf{z})\right\}=(1-\rho)$ SNR.

2) To obtain the effective throughput region of the TDM scheme, we solve the above maximization problems for different values of $\rho$ and $\tau$. More specifically, for fixed $\tau \in[0,1]$, we obtain the effective capacity pairs $\left(\mathrm{C}_{0}^{*}, \mathrm{C}_{1}^{*}\right)$ for all possible $\rho \in[0,1]$ values, and plot the rate region. We repeat the same procedure for all $\tau$ values to characterize the throughput region.

\section{Numerical Results}

First, we consider the case in which common and confidential messages are sent simultaneously. In Fig. 2, we plot the effective secrecy throughput region for two different $\left(\theta_{0}, \theta_{1}\right)$ pairs in a Rayleigh fading environment in which $z_{M}$ and $z_{E}$ are independent exponential random variables with $\mathbb{E}\left\{z_{M}\right\}=\mathbb{E}\left\{z_{E}\right\}=1$. We assume that $\gamma=1$, i.e., the noise variances at both receivers are equal. We further assume that $\mathrm{SNR}=0 \mathrm{~dB}$. In the figure, the solid-line boundary region corresponds to the case in which $\theta_{0}=\theta_{1}=0.01$. The dashed-line boundary region is for the case in which the QoS exponents are different with values $\theta_{0}=0.01$ and $\theta_{1}=0.001$. In the latter scenario, looser QoS constraints are imposed for the transmission of confidential messages. In this case, we expectedly observe that higher effective capacities for the confidential messages can be attained for fixed common message effective capacity. Hence, the effective throughput region expands.

In Fig. 3, we plot the optimal power adaptation policies $\mu_{0}(\mathbf{z})$ and $\mu_{1}(\mathbf{z})$ as a function of the channel states $\mathbf{z}=\left(z_{M}, z_{E}\right)$ for the optimal superposition strategy. In the figure, we have SNR $=-10 \mathrm{~dB}$ and $\theta_{0}=\theta_{1}=0.01$. Moreover, we assume $\lambda_{0}=0.5$, and hence these are the optimal power control policies that maximize the sum rate throughput $\mathrm{C}_{0}+\mathrm{C}_{1}$. It is obvious from the figure that the power for common message seems

$$
\mu_{0}^{*}(\mathbf{z})= \begin{cases}{\left[\frac{1}{\left.\left(\frac{\alpha_{0}}{1-\tau}\right)^{1 /\left((1-\tau) \beta_{0}+1\right)}\left(\gamma z_{E}\right)^{(1-\tau) \beta_{0} /\left((1-\tau) \beta_{0}+1\right)}-\frac{1}{\gamma z_{E}}\right]^{+},}, \quad \mathbf{z} \in \mathcal{Z}\right.} \\ {\left[\frac{1}{\left.\alpha_{0}^{1 /\left(\beta_{0}+1\right)} z_{M}^{\beta_{0} /\left(\beta_{0}+1\right)}-\frac{1}{z_{M}}\right]^{+},}\right.} & \mathbf{z} \in \mathcal{Z}^{c}\end{cases}
$$




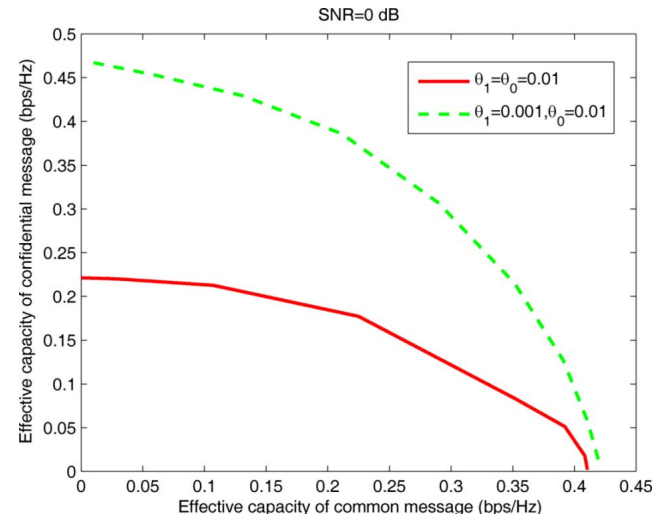

Fig. 2. Effective secrecy throughput region for two different $\left(\theta_{0}, \theta_{1}\right)$ pairs.
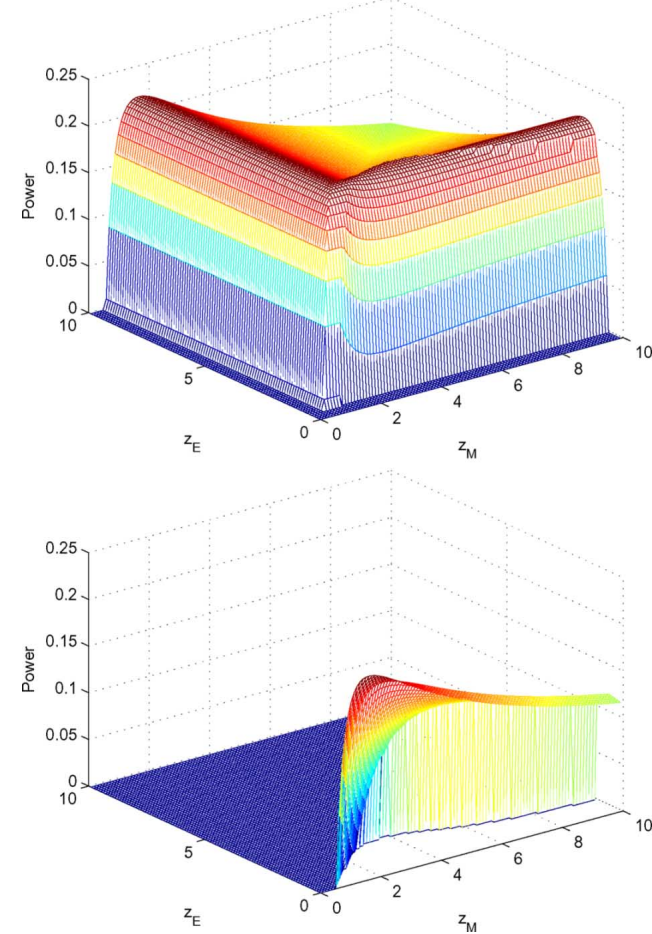

Fig. 3. Power allocated to common message and confidential message transmissions as a function of $\mathbf{z}=\left(z_{M}, z_{E}\right)$. The top figure plots the power control policy $\mu_{0}$, while the figure below plots the power control policy $\mu_{1} . \lambda_{0}=0.5$. $\mathrm{SNR}=-10 \mathrm{~dB} . \theta_{0}=\theta_{1}=0.01$

to be relatively uniformly distributed over all the entire channel state space while the power for confidential messages is concentrated in the smaller region $\mathcal{Z}$. Still, we note that the optimal $\mu_{1}$ provides relatively uniform distribution in $\mathcal{Z}$ rather than an opportunistic power allocation strategy in which more power is allocated to the transmission of confidential messages when $z_{M}$ is much larger than $z_{E}$ and less power otherwise. As will also be seen in the discussions of the following section, opportunistic power control is not necessarily optimal in the presence of buffer constraints since waiting until channel conditions get favorable may lead to buffer overflows.

In Figs. 4 and 5, we compare the optimal effective secrecy throughput region (achieved with the superposed transmission of common and confidential messages) with the one obtained using TDM as discussed in Section III-C. In Fig. 4, $\theta_{0}=0.01$ and $\theta_{1}=0.001$. The dashed line provides the boundary region of the superposition scheme. The dotted points represent the

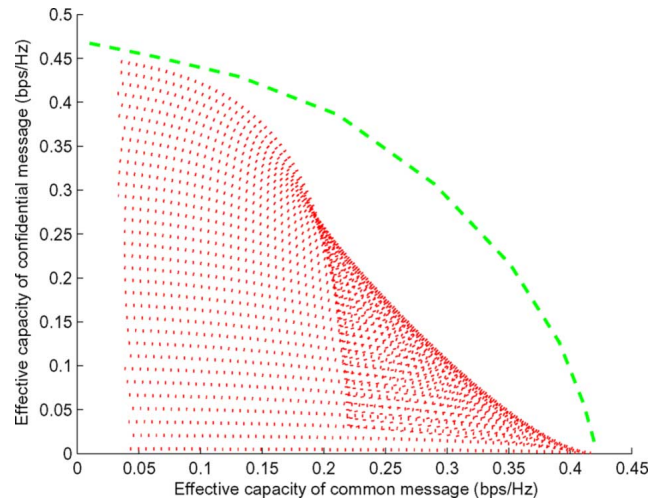

Fig. 4. Effective throughput regions for superposition and TDM schemes. $\mathrm{SNR}=0 \mathrm{~dB}, \theta_{0}=0.01$, and $\theta_{1}=0.001$. Dashed line is the boundary region of the superposition transmission. Dotted points denote the maximum effective capacity pairs $\left(\mathrm{C}_{0}^{*}, \mathrm{C}_{1}^{*}\right)$ for different values of $\tau$ and $\rho$ in the TDM scheme. Union of these points provide the effective throughput region of TDM.

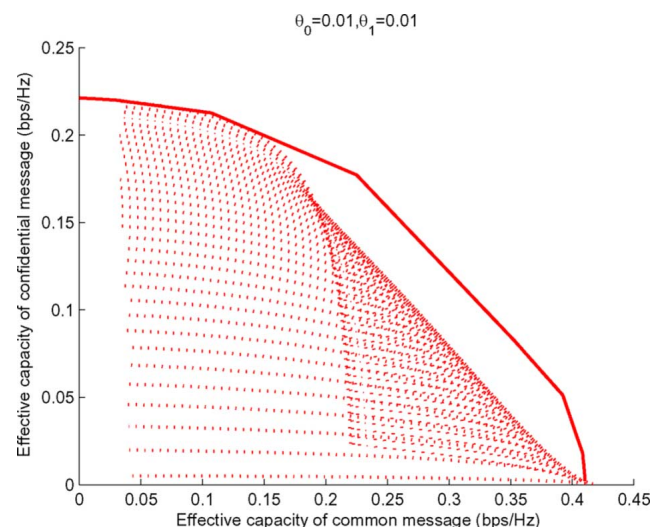

Fig. 5. Effective throughput regions for superposition and TDM schemes. SNR $=0 \mathrm{~dB}$ and $\theta_{0}=\theta_{1}=0.01$. Solid-line is the boundary region of the superposition transmission. Dotted points denote the maximum effective capacity pairs $\left(\mathrm{C}_{0}^{*}, \mathrm{C}_{1}^{*}\right)$ for different values of $\tau$ and $\rho$ in the TDM scheme. Union of these points provide the effective throughput region of TDM.

maximum effective capacity pairs $\left(\mathrm{C}_{0}^{*}, \mathrm{C}_{1}^{*}\right)$ for different values of $\tau$ and $\rho$ in the TDM scheme. The union of all the points corresponds to the effective throughput region of TDM. In the figure, we note that the region achieved with TDM is not necessarily convex. We also observe that TDM is always inside the optimal throughput region, and hence is suboptimal. However, it is interesting that when the common message effective rate is small, the TDM scheme can achieve almost the same performance as the optimal one. A similar plot with similar observations is provided in Fig. 5 for the case in which $\theta_{0}=\theta_{1}=0.01$.

\section{Effective Secure Throughrut and Optimal Power CONTROL In THE ABSENCE OF COMMON MESSAGES}

In this section, we assume that the common message rate is zero, i.e., $R_{0}=0$, and investigate the secrecy capacity and the associated optimal power control policy in the presence of QoS constraints. For this case, we identify equivalent optimization problems that are simpler to solve than the ones studied in Section III. In particular, we analyze two types of power adaptation policies. First, we consider the case in which the power control policies take into account the CSI of both the main and eavesdropper channels. Subsequently, we investigate power allocation strategies that are functions of only the CSI of the main 
channel. Additionally, we compare the power control policies obtained in this paper with the power control policies in [2] to gain further insight.

\section{A. Power Adaptation With Main and Eavesdropper Channel State Information}

In this subsection, we assume that transmitter adapts the transmitted power according to the instantaneous values of $z_{M}$ and $z_{E}$. Note that $\mu_{0}=0$ for all $\mathbf{z}$. In the absence of QoS constraints, the optimal power control policy is given by [2]

$$
\begin{aligned}
& \mu_{1}\left(z_{M}, z_{E}\right) \\
& =\frac{1}{2}\left[\sqrt{\left(\frac{1}{\gamma z_{E}}-\frac{1}{z_{M}}\right)^{2}+\frac{4}{\lambda}\left(\frac{1}{\gamma z_{E}}-\frac{1}{z_{M}}\right)}-\left(\frac{1}{z_{M}}+\frac{1}{\gamma z_{E}}\right)\right]^{+}
\end{aligned}
$$

where $[\cdot]^{+}=\max \{0, \cdot\}$, and $\lambda$ is a constant chosen to satisfies the average power constraint $\mathbb{E}\left\{\mu_{1}\left(z_{M}, z_{E}\right)\right\} \leq \mathrm{SNR}$.

In the following, we will derive the power adaptation policy by taking into account the QoS constraints. Recall that the instantaneous secrecy rate with power adaptation policy $\mu_{1}\left(z_{M}, z_{E}\right)$ is given by

$$
R_{1}= \begin{cases}\log _{2}\left(1+\mu_{1}\left(z_{M}, z_{E}\right) z_{M}\right) & \\ -\log _{2}\left(1+\gamma \mu_{1}\left(z_{M}, z_{E}\right) z_{E}\right), & \mathbf{z} \in \mathcal{Z} \\ 0, & \mathbf{z} \in \mathcal{Z}^{c}\end{cases}
$$

and the maximum effective secure throughput can be expressed as

$$
\begin{aligned}
& \mathrm{C}_{1}=\underset{\substack{\mu_{1}\left(z_{M}, z_{E}\right) \\
\mathbb{E}\left\{\mu_{1}\left(z_{M}, z_{E}\right)\right\} \leq \mathrm{SNR}}}{\max _{1} T B}-\frac{1}{\theta_{1} T B} \\
& \quad \times \log _{e}\left(\int_{0}^{\infty} \int_{0}^{\gamma z_{E}} p_{z_{M}}\left(z_{M}\right) p_{z_{E}}\left(z_{E}\right) d z_{M} d z_{E}\right. \\
& \left.+\int_{0}^{\infty} \int_{\gamma z_{E}}^{\infty}\left(\frac{1+\mu_{1}\left(z_{M}, z_{E}\right) z_{M}}{1+\gamma \mu_{1}\left(z_{M}, z_{E}\right) z_{E}}\right)^{-\beta_{1}} p_{z_{M}}\left(z_{M}\right) p_{z_{E}}\left(z_{E}\right) d z_{M} d z_{E}\right)
\end{aligned}
$$

where $p_{z_{M}}\left(z_{M}\right)$ and $p_{z_{E}}\left(z_{E}\right)$ are the probability density functions of $z_{M}$ and $z_{E}$, respectively, ${ }^{3}$ and $\beta_{1}=\theta_{1} T B / \log _{e} 2$. Note that the first term in the log function is a constant and $\log$ is a monotonically increasing function. Therefore, the maximization problem in (42) is equivalent to the following minimization

\footnotetext{
${ }^{3}$ In Section IV, we assume that $z_{M}$ and $z_{E}$ are independent. While this is not necessarily required in Section IV-A, optimal control policy results in Section IV-B depend on this assumption. Hence, we have the same assumption throughout Section IV for the sake of being consistent.
}

problem:

$$
\begin{gathered}
\min _{\substack{\mu_{1}\left(z_{M}, z_{E}\right) \\
\mathbb{E}\left\{\mu_{1}\left(z_{M}, z_{E}\right)\right\} \leq \mathrm{SNR}}} \int_{0}^{\infty} \int_{\gamma z_{E}}^{\infty}\left(\frac{1+\mu_{1}\left(z_{M}, z_{E}\right) z_{M}}{1+\gamma \mu_{1}\left(z_{M}, z_{E}\right) z_{E}}\right)^{-\beta_{1}} \\
\times p_{z_{M}}\left(z_{M}\right) p_{z_{E}}\left(z_{E}\right) d z_{M} d z_{E}
\end{gathered}
$$

It is easy to check that when $z_{M}>\gamma z_{E}$

$$
f\left(\mu_{1}\right)=\left(\frac{1+\mu_{1} z_{M}}{1+\gamma \mu_{1} z_{E}}\right)^{-\beta_{1}}
$$

is a convex function in $\mu_{1}$. Since the nonnegative weighted sum of convex functions is convex [17], we can immediately see that the objective function in (43) is also convex in $\mu_{1}$. Then, we can form the Lagrangian function, denoted as $\mathcal{J}$, shown in (45), at the bottom of the page. Taking the derivative of the Lagrangian function with respect to $\mu_{1}\left(z_{M}, z_{E}\right)$, we get the following optimality condition:

$$
\begin{aligned}
& \frac{\partial \mathcal{J}}{\partial \mu_{1}\left(z_{M}, z_{E}\right)}=\lambda-\beta_{1}\left(\frac{1+\mu_{1}\left(z_{M}, z_{E}\right) z_{M}}{1+\gamma \mu_{1}\left(z_{M}, z_{E}\right) z_{E}}\right)^{-\beta_{1}} \\
& \quad \times \frac{z_{M}-\gamma z_{E}}{\left(1+\mu_{1}\left(z_{M}, z_{E}\right) z_{M}\right)\left(1+\gamma \mu_{1}\left(z_{M}, z_{E}\right) z_{E}\right)}=0
\end{aligned}
$$

where $\lambda$ is the Lagrange multiplier whose value is chosen to satisfy the average power constraint with equality. For any channel state pairs $\left(z_{M}, z_{E}\right), \mu_{1}\left(z_{M}, z_{E}\right)$ can be obtained from the above condition. Whenever the value of $\mu_{1}\left(z_{M}, z_{E}\right)$ is negative, it follows from the convexity of the objective function with respect to $\mu_{1}\left(z_{M}, z_{E}\right)$ that the optimal value of $\mu_{1}\left(z_{M}, z_{E}\right)$ is 0 .

There is no closed-form solution to (46). However, since the right-hand side (RHS) of (46) is a monotonically increasing function, numerical techniques such as bisection search method can be efficiently adopted to derive the solution.

The secure throughput can be determined by substituting the optimal power control policy $\mu_{1}^{*}\left(z_{M}, z_{E}\right)$ in (42). Exploiting the optimality condition in (46), we can notice that when $\mu_{1}\left(z_{M}, z_{E}\right)=0$, we have $z_{M}-\gamma z_{E}=\lambda / \beta_{1}$. Meanwhile

$$
\begin{aligned}
& \left(\frac{1+\mu_{1}\left(z_{M}, z_{E}\right) z_{M}}{1+\gamma \mu_{1}\left(z_{M}, z_{E}\right) z_{E}}\right)^{-\beta_{1}} \\
& \quad \times \frac{1}{\left(1+\mu_{1}\left(z_{M}, z_{E}\right) z_{M}\right)\left(1+\gamma \mu_{1}\left(z_{M}, z_{E}\right) z_{E}\right)}<1 .
\end{aligned}
$$

Thus, we must have $z_{M}-\gamma z_{E}>\lambda / \beta_{1}$ for $\mu_{1}\left(z_{M}, z_{E}\right)>0$, i.e., $\mu_{1}\left(z_{M}, z_{E}\right)=0$ if $z_{M}-\gamma z_{E} \leq \lambda / \beta_{1}$. Hence, we can write the maximum effective secure throughput as shown in (48), at the bottom of the next page, where $\mu_{1}^{*}\left(\theta, z_{M}, z_{E}\right)$ is the derived optimal power control policy.

$$
\begin{aligned}
\mathcal{J}= & \int_{0}^{\infty} \int_{\gamma z_{E}}^{\infty}\left(\frac{1+\mu_{1}\left(z_{M}, z_{E}\right) z_{M}}{1+\gamma \mu_{1}\left(z_{M}, z_{E}\right) z_{E}}\right)^{-\beta_{1}} p_{z_{M}}\left(z_{M}\right) p_{z_{E}}\left(z_{E}\right) d z_{M} d z_{E} \\
& +\lambda\left(\int_{0}^{\infty} \int_{\gamma z_{E}}^{\infty} \mu_{1}\left(z_{M}, z_{E}\right) p_{z_{M}}\left(z_{M}\right) p_{z_{E}}\left(z_{E}\right) d z_{M} d z_{E}-\mathrm{SNR}\right)
\end{aligned}
$$




\section{B. Power Adaptation With Only Main Channel State Information}

In this section, we assume that the transmitter adapts the power level by only taking into account the CSI of the main channel (the channel between the transmitter and the legitimate receiver). When there are no QoS constraints, the optimal power control policy $\mu_{1}\left(z_{M}\right)$ is solved from [2]

$$
\begin{aligned}
& \frac{z_{M} P\left(z_{E} \leq \frac{z_{M}}{\gamma}\right)}{1+\mu_{1}\left(z_{M}\right) z_{M}} \\
& \quad-\int_{0}^{z_{M} / \gamma} \frac{\gamma z_{E}}{1+\mu_{1}\left(z_{M}\right) \gamma z_{E}} p_{z_{E}}\left(z_{E}\right) d z_{E}-\lambda=0
\end{aligned}
$$

where $\lambda$ is a constant chosen to satisfy the average power constraint $\mathbb{E}\left\{\mu_{1}\left(z_{M}, z_{E}\right)\right\} \leq \mathrm{SNR}$ with equality, and whenever the obtained $\mu_{1}\left(z_{M}\right)$ is negative, it is set to 0 .

We again investigate the optimal power control policy in the presence of QoS constraints. The instantaneous secrecy rate with power adaptation policy $\mu_{1}\left(z_{M}\right)$ is

$$
R_{1}= \begin{cases}\log _{2}\left(1+\mu_{1}\left(z_{M}\right) z_{M}\right)-\log _{2}\left(1+\gamma \mu_{1}\left(z_{M}\right) z_{E}\right), & \mathbf{z} \in \mathcal{Z} \\ 0, & \mathbf{z} \in \mathcal{Z}^{c}\end{cases}
$$

and the maximum effective secure throughput is shown in (51), at the bottom of the page. Similar to the discussion in Section IV-A, we get the following equivalent minimization problem:

$$
\begin{aligned}
\min _{\substack{\mu_{1}\left(z_{M}\right) \\
\mathbb{E}\left\{\mu_{1}\left(z_{M}\right)\right\} \leq \mathrm{SNR}}} \int_{0}^{\infty} \int_{0}^{z_{M} / \gamma}\left(\frac{1+\mu_{1}\left(z_{M}\right) z_{M}}{1+\gamma \mu_{1}\left(z_{M}\right) z_{E}}\right)^{-\beta_{1}} \\
\times p_{z_{M}}\left(z_{M}\right) p_{z_{E}}\left(z_{E}\right) d z_{E} d z_{M} .
\end{aligned}
$$

The objective function in this case is again convex, and with a similar Lagrangian optimization method, we can get the following optimality condition:

$$
\begin{aligned}
\frac{\partial \mathcal{J}}{\partial \mu_{1}\left(z_{M}\right)}= & -\beta_{1} \int_{0}^{z_{M} / \gamma}\left(\frac{1+\mu_{1}\left(z_{M}\right) z_{M}}{1+\gamma \mu_{1}\left(z_{M}\right) z_{E}}\right)^{-\beta_{1}-1} \\
& \times \frac{z_{M}-\gamma z_{E}}{\left(1+\gamma \mu_{1}\left(z_{M}\right) z_{E}\right)^{2}} p_{z_{E}}\left(z_{E}\right) d z_{E}+\lambda=0
\end{aligned}
$$

where $\lambda$ is the Lagrange multiplier whose value is chosen to satisfy the average power constraint with equality. If the obtained power level $\mu_{1}\left(z_{M}\right)$ is negative, then the optimal value of $\mu_{1}\left(z_{M}\right)$ becomes 0 according to the convexity of the objective function in (52). The RHS of (53) is still a monotonic increasing function of $\mu_{1}\left(z_{M}\right)$.

The secure throughput can be determined by substituting the optimal power control policy $\mu_{1}^{*}\left(z_{M}\right)$ in (51). Exploiting the optimality condition in (53), we notice that when $\mu_{1}\left(z_{M}\right)=0$, we have

$$
\begin{aligned}
-\beta_{1} \int_{0}^{z_{M} / \gamma}\left(z_{M}-\gamma z_{E}\right) p_{z_{E}}\left(z_{E}\right) d z_{E}+\lambda & =0 \\
\Rightarrow \int_{0}^{z_{M}} P\left(z_{E} \leq \frac{t}{\gamma}\right) d t & =\frac{\lambda}{\beta_{1}} .
\end{aligned}
$$

Let us denote the solution to the above equation as $\alpha$. Considering that

$$
\left(\frac{1+\mu_{1}\left(z_{M}\right) z_{M}}{1+\gamma \mu_{1}\left(z_{M}\right) z_{E}}\right)^{-\beta_{1}-1} \frac{1}{\left(1+\gamma \mu_{1}\left(z_{M}\right) z_{E}\right)^{2}}<1
$$

we must have $z_{M}>\alpha$ for $\mu_{1}\left(z_{M}\right)>0$, i.e., $\mu_{1}\left(z_{M}\right)=0$ if $z_{M} \leq \alpha$. Hence, we can write the maximum effective secure throughput as shown in (57), at the bottom of the page, where $\mu_{1}^{*}\left(z_{M}\right)$ is the derived optimal power control policy.

$$
\begin{aligned}
\mathrm{C}_{1}=-\frac{1}{\theta_{1} T B} \log _{e} & \left(\int_{0}^{\infty} \int_{0}^{\gamma z_{E}+\lambda / \beta_{1}} p_{z_{M}}\left(z_{M}\right) p_{z_{E}}\left(z_{E}\right) d z_{M} d z_{E}+\int_{0}^{\infty} \int_{\gamma z_{E}+\lambda / \beta_{1}}^{\infty}\left(\frac{1+\mu_{1}^{*}\left(z_{M}, z_{E}\right) z_{M}}{1+\gamma \mu_{1}^{*}\left(z_{M}, z_{E}\right) z_{E}}\right)^{-\beta_{1}}\right. \\
& \left.\times p_{z_{M}}\left(z_{M}\right) p_{z_{E}}\left(z_{E}\right) d z_{M} d z_{E}\right)
\end{aligned}
$$

$$
\begin{aligned}
\mathrm{C}_{1}= & \underset{\substack{\mu_{1}\left(z_{M}\right) \\
\mathbb{E}\left\{\mu_{1}\left(z_{M}\right)\right\} \leq \mathrm{SNR}}}{\max _{1} T B}-\frac{1}{\theta_{1} T B} \\
& \times \log _{e}\left(\int_{0}^{\infty} \int_{z_{M} / \gamma}^{\infty} p_{z_{M}}\left(z_{M}\right) p_{z_{E}}\left(z_{E}\right) d z_{E} d z_{M}+\int_{0}^{\infty} \int_{0}^{z_{M} / \gamma}\left(\frac{1+\mu_{1}\left(z_{M}\right) z_{M}}{1+\gamma \mu_{1}\left(z_{M}\right) z_{E}}\right)^{-\beta_{1}} p_{z_{M}}\left(z_{M}\right) p_{z_{E}}\left(z_{E}\right) d z_{E} d z_{M}\right)
\end{aligned}
$$

$$
\begin{aligned}
\mathrm{C}_{1}=-\frac{1}{\theta_{1} T B} \log _{e}\left(\int_{0}^{\alpha} \int_{0}^{\infty} p_{z_{M}}\left(z_{M}\right) p_{z_{E}}\left(z_{E}\right) d z_{E} d z_{M}+\int_{\alpha}^{\infty} \int_{z_{M} / \gamma}^{\infty} p_{z_{M}}\left(z_{M}\right) p_{z_{E}}\left(z_{E}\right) d z_{E} d z_{M}\right. \\
\left.+\int_{\alpha}^{\infty} \int_{0}^{z_{M} / \gamma}\left(\frac{1+\mu_{1}^{*}\left(z_{M}\right) z_{M}}{1+\gamma \mu_{1}^{*}\left(z_{M}\right) z_{E}}\right)^{-\beta_{1}} \times p_{z_{M}}\left(z_{M}\right) p_{z_{E}}\left(z_{E}\right) d z_{E} d z_{M}\right)
\end{aligned}
$$




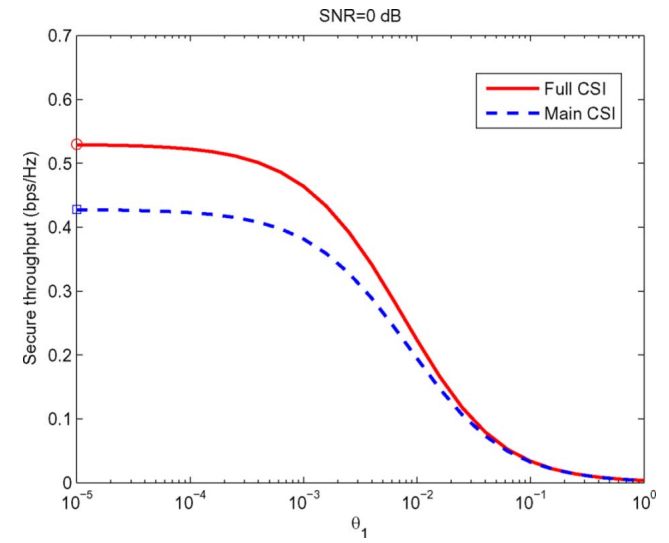

Fig. 6. Effective secure throughput versus $\theta_{1}$ in the Rayleigh fading channel with $\mathbb{E}\left\{z_{E}\right\}=\mathbb{E}\left\{z_{M}\right\}=1 \cdot \gamma=1$.

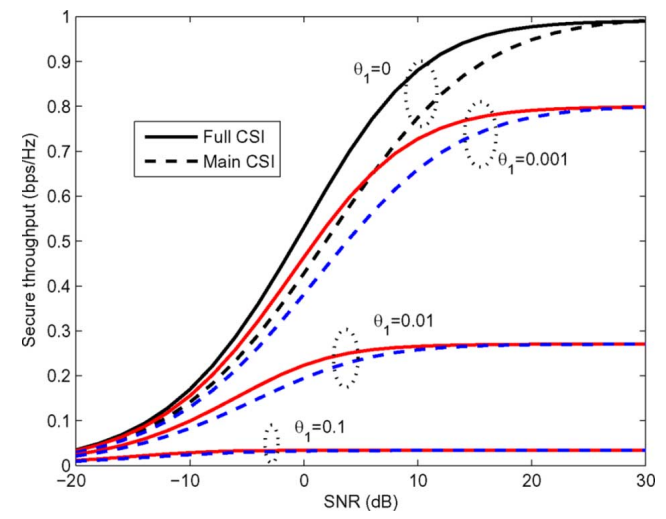

Fig. 7. Effective secure throughput versus SNR in the Rayleigh fading channel with $\mathbb{E}\left\{z_{E}\right\}=\mathbb{E}\left\{z_{M}\right\}=1 \cdot \gamma=1$.

\section{Numerical Results}

In Fig. 6, we plot the effective secure throughput as a function of the QoS exponent $\theta_{1}$ in the Rayleigh fading channel with $\gamma=1$ when the power is adapted with respect to the full CSI (i.e., the CSI of main and eavesdropper channels) and also with respect to only the main CSI. It can be seen from the figure that as the $\mathrm{QoS}$ constraints become more stringent and hence as the value of $\theta_{1}$ increases, little improvement is provided by considering the CSI of the eavesdropper channel in the power adaptation. In Fig. 7, we plot the effective secure throughput as SNR varies for $\theta_{1}=\{0,0.001,0.01,0.1\}$. Note that $\theta_{1}=0$ represents the case in which there are no QoS constraints, and the optimal power control policies are given in (40) and (49), respectively. Not surprisingly, we again observe that taking into account the CSI of the eavesdropper channel in the power adaptation policy does not provide many gains in terms of increasing the effective secure throughput in the large SNR regime. Also, as QoS constraints become more strict, we similarly note that adapting the power with full CSI does not increase the rate of secure transmission much even at medium SNR levels.

To characterize the power allocation strategy, we plot in Fig. 8 the power distribution as a function of $\left(z_{M}, z_{E}\right)$ for the full CSI case when $\theta_{1}=0.01$ and $\theta_{1}=0$. In the figure, we see that for both values of $\theta_{1}$, no power is allocated for transmission when $z_{M}<z_{E}$ which is expected under the assumption of equal noise powers, i.e., $N_{1}=N_{2}$. We note that when $\theta_{1}=0$ and hence there are no buffer constraints, the power control policy as derived in [2]

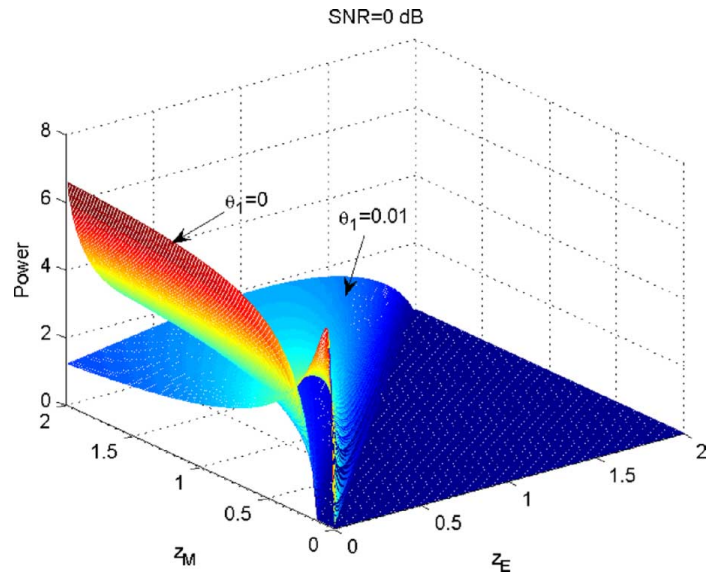

Fig. 8. Power allocation for the full CSI scenario with SNR $=0 \mathrm{~dB}$ in the Rayleigh fading channel with $\mathbb{E}\left\{z_{E}\right\}=\mathbb{E}\left\{z_{M}\right\}=1 \cdot \gamma=1$.

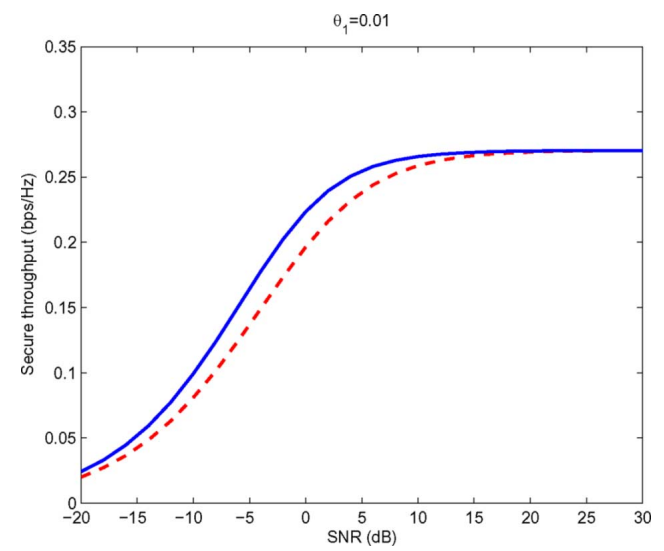

Fig. 9. Effective secure throughput versus SNR in the Rayleigh fading channel with $\mathbb{E}\left\{z_{E}\right\}=\mathbb{E}\left\{z_{M}\right\}=1 . \gamma=1$. The solid-line plots the optimal secure throughput achieved by the optimal power control policy with full CSI. Dashed line provides the secure throughput achieved by the suboptimal power control policy given in (40). $\theta_{1}=0.01$.

is more like opportunistic transmission policy. More power is allocated for cases in which the difference $z_{M}-z_{E}$ is large. Therefore, the transmitter favors the times at which the main channel is much better than the eavesdropper channel. At these times, the transmitter sends the information at a high rate with large power. When $z_{M}-z_{E}$ is small, transmission occurs at a small rate with small power. However, this strategy is clearly not optimal in the presence of buffer constraints because waiting to transmitat a high rate until the main channel becomes much stronger than the eavesdropper channel can lead to buildup in the buffer and incur large delays. Hence, we do not observe this opportunistic transmission strategy when $\theta=0.01$. In this case, we note that a more uniform powerallocation is preferred. In ordernot to violate the limitations on the buffer length, transmission at a moderate power level is performed even when $z_{M}-z_{E}$ is small.

Finally, we analyze the impact of taking the QoS constraints into account in the power control policies. In Fig. 9, we set $\theta_{1}=0.01$ and we plot the effective secure throughput attained by employing the optimal power control (solid line) and the suboptimal power control in (40) (dashed line) which does not take into consideration the presence of QoS limitations and is optimal only when $\theta_{1}=0$. We observe that optimal power control strategy is especially beneficial in the SNR range from -10 to $10 \mathrm{~dB}$, and the performance gap again vanishes for very high SNR values. 


\section{CONCLUSION}

In this paper, we have investigated the fading broadcast channels with confidential messages under statistical QoS constraints. We have first defined the effective secure throughput region and proven the convexity of this region. Then, optimal power control policies that achieve the points on the boundary of the throughput region are investigated. We have determined the conditions satisfied by the optimal power control policies. In particular, we have identified an algorithm for computing the optimal power allocated to each fading state using the optimality conditions. Additionally, we have analyzed the effective throughput region achieved through TDM of common and confidential messages. We have found that while being strictly suboptimal, TDM performance can approach that of the superposition strategies when the common message effective rate is low. When the broadcast channel is reduced to the wiretap channel with zero common message rate, we have investigated two types of optimal power allocation policies that maximize the effective secure throughput. In particular, we have noted that the transmitter allocates power more uniformly instead of concentrating its power for the cases in which the main channel is much stronger than the eavesdropper channel. By numerically comparing the obtained effective secure throughput, we have shown that as QoS constraints become more stringent, the benefits of incorporating the CSI of the eavesdropper channel in the power control policy diminish.

\section{REFERENCES}

[1] A. D. Wyner, "The wire-tap channel," Bell Syst. Tech. J., vol. 54, pp. 1355-1387, Oct. 1975.

[2] P. Gopala, L. Lai, and H. El Gamal, "On the secrecy capacity of fading channels," IEEE Trans. Inf. Theory, vol. 54, no. 10, pp. 4687-4698, Oct. 2008.

[3] Y. Liang, H. V. Poor, and S. Shamai, "Secure communication over fading channels," IEEE Trans. Inf. Theory, vol. 54, no. 6, pp. 2470-2492, Jun. 2008.

[4] E. Ekrem and S. Ulukus, "Secrecy capacity of a class of broadcast channels with an eavesdropper," Special Isssue on Wireless Physical Layer Security, EURASIP J. Wireless Commun. Netw., vol. 2009, pp. 1:1-1:29, Mar. 2009, Article 1.

[5] R. Liu, I. Maric, P. Spasojevic, and R. D. Yates, "Discrete memoryless interference and broadcast channels with confidential messages: Secrecy rate regions," Special Issue on Information Theoretic Security, IEEE Trans. Inf. Theory, vol. 54, no. 6, pp. 2493-2507, Jun. 2008.

[6] D. Wu and R. Negi, "Effective capacity: A wireless link model for support of quality of service," IEEE Trans. Wireless Commun., vol. 2, no. 4, pp. 630-643, Jul. 2003.

[7] J. Tang and X. Zhang, "Quality-of-service driven power and rate adaptation over wireless links," IEEE Trans. Wireless Commun., vol. 6, no. 8, pp. 3058-3068, Aug. 2007.

[8] J. Tang and X. Zhang, "Cross-layer-model based adaptive resource allocation for statistical QoS guarantees in mobile wireless networks," IEEE Trans. Wireless Commun., vol. 7, no. 6, pp. 2318-2328, Jun. 2008.

[9] L. Liu, P. Parag, J. Tang, W.-Y. Chen, and J.-F. Chamberland, "Resource allocation and quality of service evaluation for wireless communication systems using fluid models," IEEE Trans. Inf. Theory, vol. 53, no. 5, pp. 1767-1777, May 2007.

[10] M. C. Gursoy, D. Qiao, and S. Velipasalar, "Analysis of energy efficiency in fading channel under QoS constrains," IEEE Trans. Wireless Commun., vol. 8, no. 8, pp. 4252-4263, Aug. 2009.

[11] D. Qiao, M. C. Gursoy, and S. Velipasalar, "The impact of QoS constraints on the energy efficiency of fixed-rate wireless transmissions," IEEE Trans. Wireless Commun., vol. 8, no. 12, pp. 5957-5969, Dec. 2009.
[12] Y. Liang, H. V. Poor, and L. Ying, "Wireless broadcast networks: Reliability, security and stability," in Proc. Information Theory and Applications Workshop (ITA), Jan. 2008, pp. 249-255.

[13] M. Youssef, H. El Gamal, K. Khalil, and O. O. Koyluoglu, "On the delay limited secrecy capacity of fading channels," in Proc. IEEE Int. Symp. Information Theory (ISIT) 2009, Jun./Jul. 28-3, 2009, pp. 2617-2621.

[14] D. Qiao, M. C. Gursoy, and S. Velipasalar, "Secure communication over fading channels with statistical QoS constraints," in Proc. IEEE Int. Symp. Information Theory (ISIT 2010), Jun. 13-18, 2010, pp. 2503-2507.

[15] D. Qiao, M. C. Gursoy, and S. Velipasalar, "Secure broadcasting over fading Channels with statistical QoS constraints," in Proc. IEEE Global Telecommunications Conf. (Globecom 2010), Miami, FL, Dec. 6-10, 2010.

[16] A. Goldsmith, Wireless Communications, 1st ed. Cambridge, U.K.: Cambridge Univ. Press, 2005.

[17] S. Boyd and L. Vandenberghe, Convex Optimization. Cambridge, U.K.: Cambridge Univ. Press, 2004.

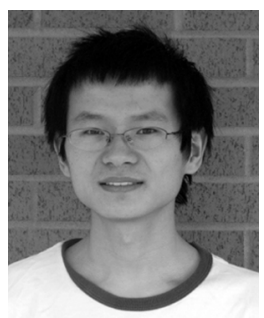

Deli Qiao (S'09) received the B.E. degree in electrical engineering from Harbin Institute of Technology, Harbin, China, in 2007. He is currently a research assistant working toward the Ph.D. degree in the Department of Electrical Engineering, University of Nebraska-Lincoln, Lincoln, NE.

His research interests include information theory and wireless communications, with an emphasis on quality of service (QoS) provisioning.

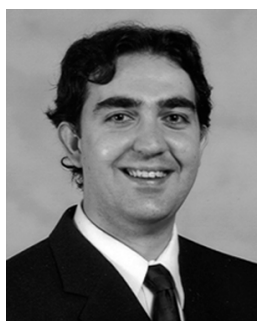

Mustafa Cenk Gursoy (S'01-M'04) received the B.S. degree in electrical and electronics engineering from Bogazici University, Istanbul, Turkey, in 1999, with high distinction, and the Ph.D. degree in electrical engineering from Princeton University, Princeton, NJ, in 2004.

In the summer of 2000 , he worked at Lucent Technologies, Holmdel, NJ, where he conducted performance analysis of DSL modems. Since September 2004, he has been with the Department of Electrical Engineering at the University of Nebraska-Lincoln (UNL), Lincoln, NE, where he is currently an Associate Professor. His research interests are in the general areas of wireless communications, information theory, communication networks, and signal processing. $\mathrm{He}$ is currently a member of the editorial boards of IEEE TRANSACTIONS ON Wireless COMMUNiCATIONS AND PHYSICAL COMMUNICATION (Elsevier).

Dr. Gursoy was a recipient of the Gordon Wu Graduate Fellowship from Princeton University between 1999 and 2003. He received an NSF CAREER Award in 2006. More recently, he received the EURASIP Journal of Wireless Communications and Networking Best Paper Award, the UNL College Distinguished Teaching Award, and the Maude Hammond Fling Faculty Research Fellowship.

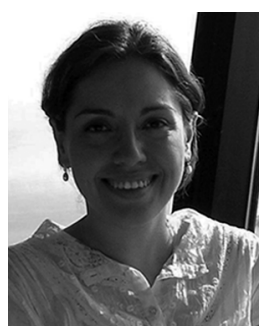

Senem Velipasalar (S'04-M'06) received the B.S. degree in electrical and electronic engineering with high honors from Bogazici University, Turkey, in 1999, the M.S. degree in electrical sciences and computer engineering from Brown University, in 2001, and the M.A. and Ph.D. degrees in electrical engineering from Princeton University in 2004 and 2007, respectively.

She is currently an assistant professor in the Department of Electrical Engineering at the University of Nebraska-Lincoln (UNL), Lincoln, NE. Her research interests include computer vision, video/image processing, distributed smart camera systems, pattern recognition, statistical learning, and signal processing. During the summers of 2001 through 2005, she worked in the Exploratory Computer Vision Group at IBM T. J. Watson Research Center.

Dr. Velipasalar is a recipient of the IBM Patent Application Award, and Princeton and Brown University Graduate Fellowships. She received the Best Student Paper Award at the IEEE International Conference on Multimedia \& Expo (ICME) in 2006. 